\title{
Survival outcomes of neoadjuvant and adjuvant chemoradiotherapy for locally advanced adenocarcinoma of the oesophagogastric junction: a retrospective cohort study using the SEER database
}

\author{
Fan Zhang ${ }^{1} \wedge$, Xingyu Feng ${ }^{2}$, Yong $\mathrm{Li}^{2}$, Juan Yan $^{3}$, Zhilin Zhang ${ }^{4}$, Xiao Song ${ }^{4}$ \\ ${ }^{1}$ School of Graduate Studies, Hebei North University, Zhangjiakou, China; ${ }^{2}$ Department of General Surgery, Guangdong Provincial People's \\ Hospital, Guangzhou, China; ${ }^{3}$ Department of Pharmacy, the First Affiliated Hospital of Hebei North University, Zhangjiakou, China; ${ }^{4}$ Department \\ of Radiotherapy, the First Affiliated Hospital of Hebei North University, Zhangjiakou, China \\ Contributions: (I) Conception and design: X Feng; (II) Administrative support: X Song, Z Zhang; (III) Provision of study materials or patients: Y Li; \\ (IV) Collection and assembly of data: F Zhang; (V) Data analysis and interpretation: F Zhang, J Yan; (VI) Manuscript writing: All authors; (VII) Final \\ approval of manuscript: All authors. \\ Correspondence to: Dr. Xiao Song. The First Affiliated Hospital of Hebei North University, No. 12 Changqing Road, Qiaoxi District, Zhangjiakou, \\ China. Email: congcongdad@163.com.
}

\begin{abstract}
Background: Both neoadjuvant chemoradiotherapy (nCRT) and adjuvant chemoradiotherapy (aCRT) have survival advantages over surgery alone in patients with adenocarcinoma of the oesophagogastric junction (AEG). However, whether there is a difference in the survival benefit between these two treatments and who can benefit from them remains controversial, and there are currently no randomised controlled trials to address these issues. This study compared the survival outcomes of patients with locally advanced AEG receiving nCRT and aCRT.
\end{abstract}

Methods: The data of patients with locally advanced AEG were collected from the Surveillance, Epidemiology, and End Results (SEER) database (2004-2015). Patients in the nCRT and aCRT groups were propensity-score matched 1:1, and the Kaplan-Meier method and log-rank test were used for survival analysis between the two groups. Univariable and multivariable Cox regression models were performed to identify the prognostic factors.

Results: Of the 1,436 cases diagnosed as locally advanced AEG, we included 442 in the final analysis. The median overall survival (OS) of the nCRT and aCRT cohorts were 30.0 and 25.0 months, respectively $(\mathrm{P}=0.042)$, and the median tumour specific survival times were 37.0 and 31.0 months, respectively $(\mathrm{P}=0.249)$. Multivariable Cox regression analysis showed that OS was independently related to age $[<60$ years $v s$. $\geq 70$ years, hazard ratio $(\mathrm{HR})=0.619,95 \% \mathrm{CI}$ : 0.510-0.751, $\mathrm{P}<0.001 ; 60-69$ years $v s . \geq 70$ years, $\mathrm{HR}=0.661$, 95\% CI: $0.536-0.814, \mathrm{P}<0.001]$ and $\mathrm{N}$ stage (N2 vs. N1, HR =1.213, 95\% CI: 1.002-1.468, $\mathrm{P}=0.048$; N3 vs. $\mathrm{N} 1, \mathrm{HR}=1.606,95 \% \mathrm{CI}: 1.190-2.167, \mathrm{P}=0.002)$. Through stratifying patients by TNM stage, stage IIIB, and N1 stage, we observed that patients receiving nCRT had a better prognosis.

Conclusions: Patients receiving nCRT had significantly better survival than those receiving aCRT. nCRT may offer some therapeutic benefits in patients with IIIB stage AEG.

Keywords: Adenocarcinoma of the oesophagogastric junction (AEG); neoadjuvant chemoradiotherapy (nCRT); adjuvant chemoradiotherapy (aCRT); propensity score matching (PSM) analysis; Surveillance, Epidemiology, and End Results (SEER) database

^ ORCID: 0000-0001-9274-1284. 
Submitted Nov 02, 2021. Accepted for publication Dec 30, 2021.

doi: 10.21037/jgo-21-815

View this article at: https://dx.doi.org/10.21037/jgo-21-815

\section{Introduction}

Gastric cancer is the fifth most common malignancy and the fourth leading cause of cancer-related mortality (1). Although the incidence of distal gastric cancer has been decreasing steadily worldwide, the incidence of adenocarcinoma of the oesophagogastric junction (AEG) has been increasing annually (2-4). The study shows that the proportion of AEG in gastric cancer is $22.3 \%$ during in the $1988-1992$ years, which has increased to $35.7 \%$ in 2008-2012 years (5).

AEG is defined as adenocarcinoma within $5 \mathrm{~cm}$ above or below the oesophagogastric junction (EGJ) (6). AEG is divided into three types based on the distance between the main part of the tumour and the dentate line. Tumours whose centre is located $1-5 \mathrm{~cm}$ above the EGJ with intestinal metaplasia or Barrett's oesophagus are classified as distal oesophageal adenocarcinoma or type I. Cardiac carcinomas located $1 \mathrm{~cm}$ above to $2 \mathrm{~cm}$ below the EGJ are known as type II. Type III tumours are defined as those located $2-5 \mathrm{~cm}$ below the EGJ, and those extending to the EGJ and are also known as subcardial carcinomas (7-9). This classification provides a theoretical standard for surgical selection worldwide.

Locally advanced AEG is defined as pathological stage T2-4a, N1-3, M0, and radical resection should be performed for such tumours in clinical evaluation (10). However, the 5 -year survival rate after radical resection remains low $(11,12)$. Therefore, there is an urgent need for additional treatment methods to improve survival. At present, the treatment of AEG is mainly a surgery-based comprehensive treatment mode, including perioperative chemotherapy, neoadjuvant chemoradiotherapy (nCRT), neoadjuvant chemotherapy, and adjuvant chemoradiotherapy (aCRT). Many studies have shown that compared with simple surgery, the above comprehensive treatment mode has achieved significant survival benefits, for examples, the COSS trial (13) established the status of nCRT and the INT0116 trial $(14,15)$ in the United States also showed the therapeutic advantages of aCRT. However, some experimental studies have shown that nCRT does not show survival benefits compared with neoadjuvant chemotherapy (16). Therefore, there is still no unified standard for the adjuvant treatment of AEG, and physicians in the United States, Europe, and Asia have different standards.

These studies generally have the following defects. Firstly, there are currently no randomized controlled trials comparing nCRT and aCRT, and it is not clear which patients might benefit more from nCRT or aCRT. In addition, previous studies on adjuvant treatment modes were indirect comparison, while this study directly compared the two treatment modes to explore whether there were benefit differences. Furthermore, Previous studies have combined esophagogastric junction adenocarcinoma with esophageal cancer or gastric cancer, but the therapeutic sensitivity of adenocarcinoma and squamous cell carcinoma is quite different. By contrast, all patients included in this study are patients with AEG.

The Surveillance, Epidemiology, and End Results (SEER) database covers about $35 \%$ of the U.S. population and contains the information of primary tumor location, morphology, stage, treatment and follow-up of millions of tumor patients in multiple state and county multi center medical institutions in the United States, which can avoid the selection bias of single institution and sick population. In summary, this study used the SEER database to conduct 1:1 propensity score matching (PSM) analysis to compare the survival outcomes of patients with locally advanced AEG receiving nCRT and aCRT. We present the following article in accordance with the STROBE reporting checklist (available at https://jgo.amegroups.com/article/ view/10.21037/jgo-21-815/rc).

\section{Methods}

\section{Patient selection}

Our study is a retrospective cohort study and the case origin is the SEER database published on August 8, 2019 (named Nov 2018 Sub Set from 1975 to 2016), and the access right and usufruct right of SEER database were officially granted (according to login ID 16865-Nov2019). The clinicopathological and follow-up data of AEG patients were collected using SEER*Stat v8.3.8 software, including surgical and chemoradiotherapy data. The number of cases meeting the inclusion criteria during the study period determined the sample size. Finally, after removing the missing values for 


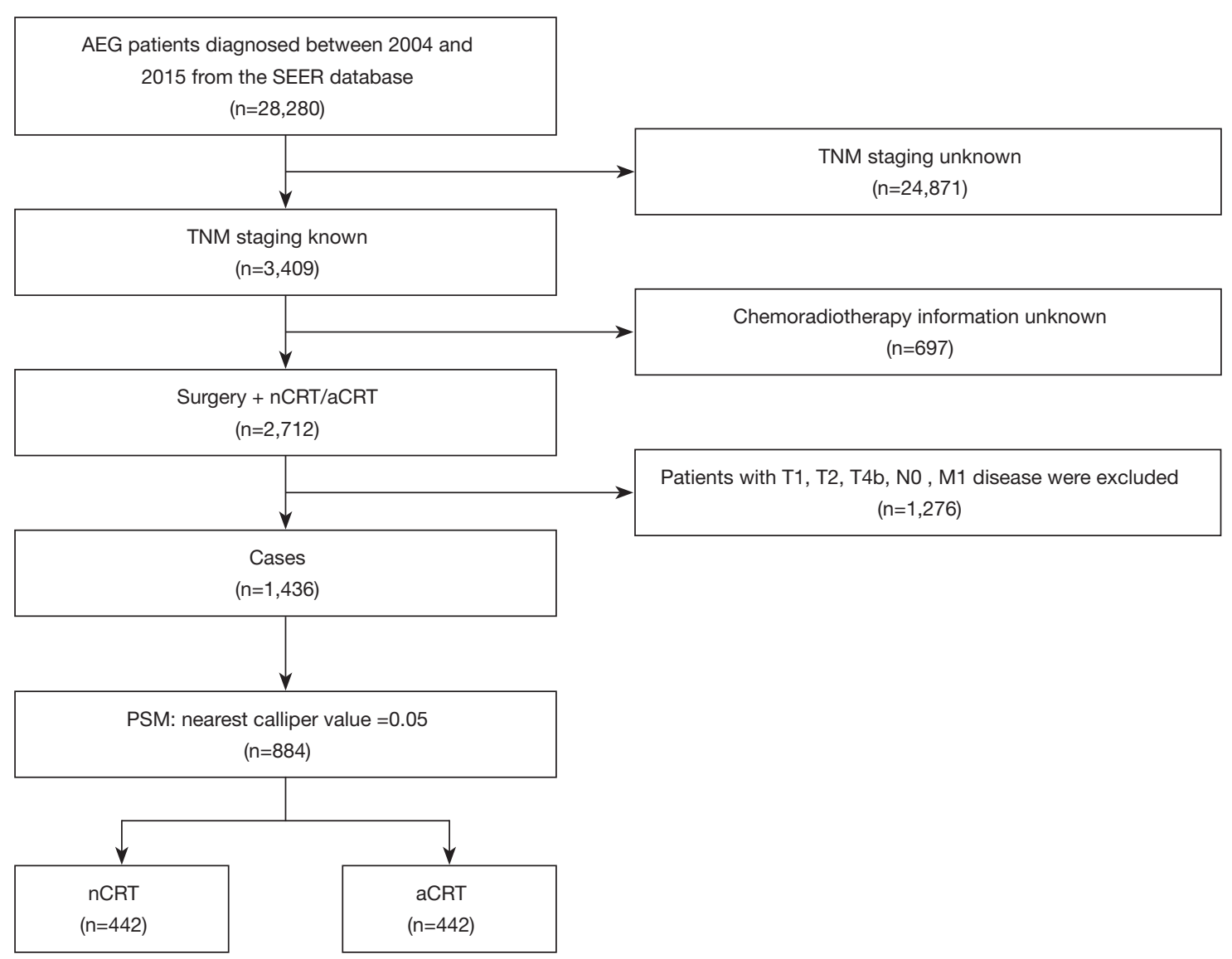

Figure 1 Patient selection flow chart. AEG, adenocarcinoma of the oesophagogastric junction; SEER, Surveillance, Epidemiology, and End Results; TNM, TNM stage; nCRT, neoadjuvant chemoradiotherapy; aCRT, adjuvant chemoradiotherapy; PSM, propensity score matching.

the relevant variables in the study, 1,436 patients diagnosed with locally advanced AEG from 2004 to 2015 were selected, and the data was complete. The follow-up deadline is the database release time. The inclusion criteria were as follows: (I) patients diagnosed with AEG only; (II) those confirmed as T3-4a, N+, M0; (III) patients who underwent surgery; (IV) cases with information on neoadjuvant and adjuvant therapy; (V) patients with a known cause of death; (VI) cases involving a histological diagnosis; and (VII) complete clinical and follow-up information and delete the missing values that will significantly affect the study. The specific data filtering process is displayed in Figure 1. The study was conducted in accordance with the Declaration of Helsinki (as revised in 2013).

\section{Statistical analysis}

IBM SPSS Statistical software 26.0 software (IBM,
Armonk, NY, USA) and R version 4.0.5 software (https:// www.r-project.org/) were utilized for statistical analysis. $\mathrm{P}<0.05$ was considered to indicate a statistically significant difference.

The baseline data were balanced by PSM, with a caliper value of 0.05 . This study adopted the nearest neighbor matching method in which substitution is not allowed and the patient is matched only once, and established a logistic regression model that could predict the possibility of receiving nCRT or aCRT, which was applied for the propensity score. Age, race, sex, grade, T stage, N stage, and total stage were utilized for matching. The standardized mean differences before and after matching are illuminated in Figure 2. The balance between datum line covariates in both the matched and unmatched cohorts was scanned by standardized differences, and $<10 \%$ was adequately credible (17). Next, we used the chi square test to compare patient and tumour characteristics in the pre- 


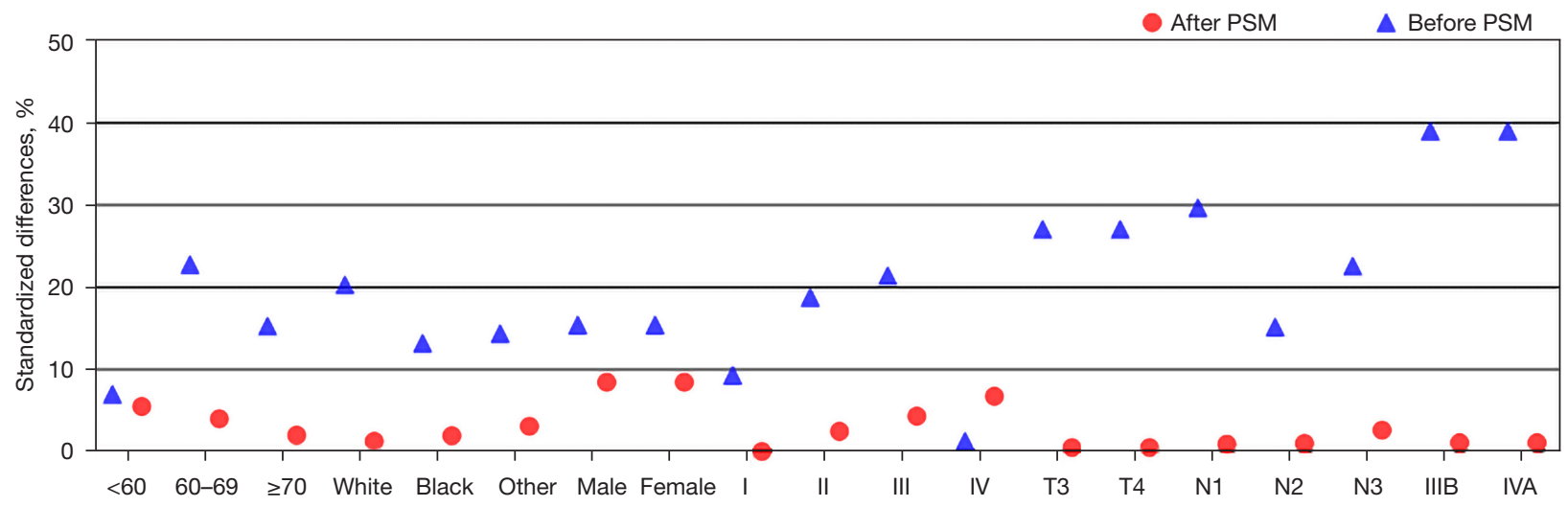

Figure 2 Standardized differences before and after PSM. PSM, propensity score matching.

and post-matched cohorts.

The main end-points of this study were overall survival (OS) and cancer-specific survival (CSS). OS was the period between diagnosis of AEG and death from any cause, while CSS refers to the period between diagnosis of AEG and death due to AEG. The survival rates of AEG patients treated with nCRT and aCRT groups after matching were investigated by Kaplan-Meier analysis with the logrank test. Univariable and multivariable Cox proportional hazards regression was applied to investigate the independent survival predictors for locally advanced AEG patients. Meanwhile, we stratified the patients according to tumor node metastasis classification (TNM).

\section{Results}

\section{Baseline characteristics}

In total, 1,436 of 28,280 AEG patients during the period 2004 to 2015 met the inclusion criteria. Of these patients, $894(62.3 \%)$ received nCRT and 542 (37.7\%) received aCRT (Table 1). Interestingly, since 2012, more patients received nCRT than aCRT (Figure 3). Following PSM analysis, 884 patients were included in the nCRT and aCRT cohorts, respectively. In the matched cohort, the division of baseline characteristics was balanced (Table 2).

\section{Impact of nCRT and aCRT on OS}

After Kaplan-Meier calculation of OS, there was a noteworthy diversity in survival between patients who underwent nCRT and those who underwent aCRT (logrank $\mathrm{P}=0.042$ ) (Figure $4 A$ ). The median $\mathrm{OS}$ was 30.0 months
(95\% CI: 25.6-34.4 months) in patients receiving nCRT and 25.0 months (95\% CI: 22.5-27.5 months) in patients receiving aCRT. The 3 -year OS rate was $43.5 \%$ for all patients receiving $\mathrm{nCRT}$, and $38.5 \%$ for those receiving aCRT. Univariate Cox regression analysis demonstrated that OS was markedly correlated with age, $\mathrm{N}$ stage, TNM stage, and treatment group.

Next, these meaningful variables were all fitted into the multivariate Cox regression analysis. After amending for these meaningful variables by multivariate Cox regression analysis, nCRT was found to not a protective factor for OS of AEG patients [hazard ratio (HR), 1.151; 95\% CI: 0.978 1.355, $\mathrm{P}=0.091$; Table 3]. However, age and $\mathrm{N}$ stage were independent predictive factors of OS.

\section{Impact of nCRT and aCRT on CSS}

The log-rank test $(\mathrm{P}=0.249)$ showed no significant differences in CSS between the two cohorts (Figure 4B). The median CSS was 37.0 months (95\% CI: 29.0-45.0 months) in patients undergoing nCRT, and 31.0 months (95\% CI: 24.3-37.7 months) for receiving aCRT. The 3-year CSS rate for patients receiving nCRT was $50.2 \%$, and $47.1 \%$ for those receiving aCRT. In the univariable Cox proportional hazards regression analysis, TNM stage and $\mathrm{N}$ stage were found to be related to CSS, and were included in the multivariate Cox proportional hazard regression model. After multivariable analysis, nCRT was shown to not be a statistically significant protective factor for CSS (HR, 1.091; 95\% CI: 0.907-1.313, P=0.353; Table 4). Nevertheless, it was demonstrated that the TNM stage covariates were unique influencing factors for CSS. 
Table 1 Patient characteristics before matching

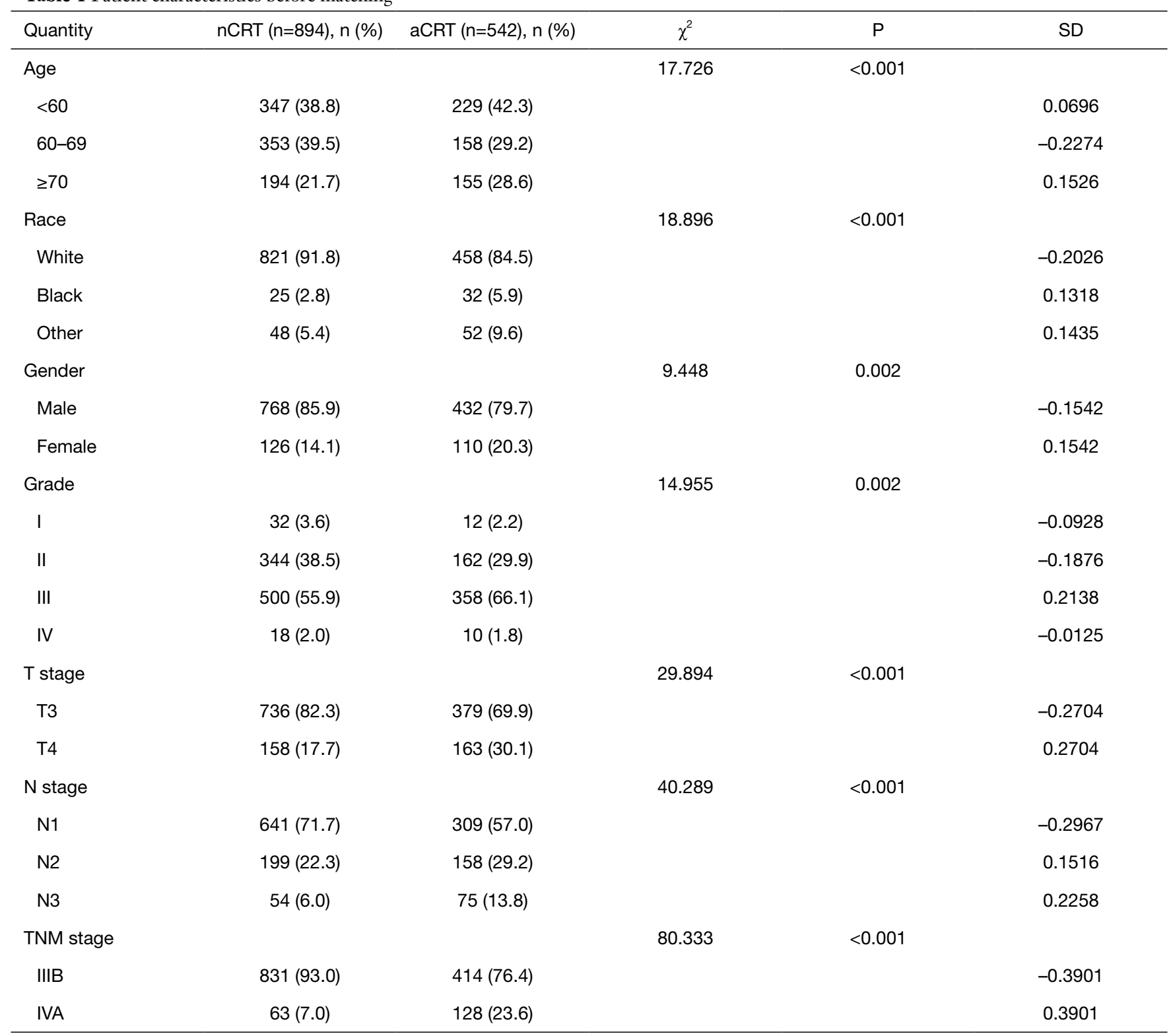

The Chi square test was applied to contrast patient and cancer characteristics in the pre-matched cohort. Statistical significance was set at $\mathrm{P}<0.05$. $\mathrm{nCRT}$, neoadjuvant chemoradiotherapy; aCRT, adjuvant chemoradiotherapy; SD, standardized differences.

\section{Survival outcomes stratified by $T$ stage, $N$ stage, and TNM stage}

Total stage-based subgroup analysis showed that a notable survival benefit was reflected in patients receiving nCRT (Figure 5), especially the OS in IIIB stage subgroup (Figure $5 A$ ), but there were no differences in CSS (Figure $5 B$ ). However, this effect was not prominent neither in OS nor in CSS among patients with stage IVA stage
(Figure 5C,5D). In the matched cohort, patients with stage IIIB disease who received nCRT had a more favourable OS than those who received aCRT (log-rank $\mathrm{P}=0.026)$. The median OS was 31.0 months (95\% CI: 26.0-36.0 months) in patients receiving nCRT, but was only 26.0 months (95\% CI: 23.3-28.7 months) in patients receiving aCRT.

Subsequently, patients were stratified based on the $\mathrm{N}$ stage. Among patients with N1 disease, those who received nCRT had the best OS (log-rank test, $\mathrm{P}=0.014$ ) (Figure 6). 


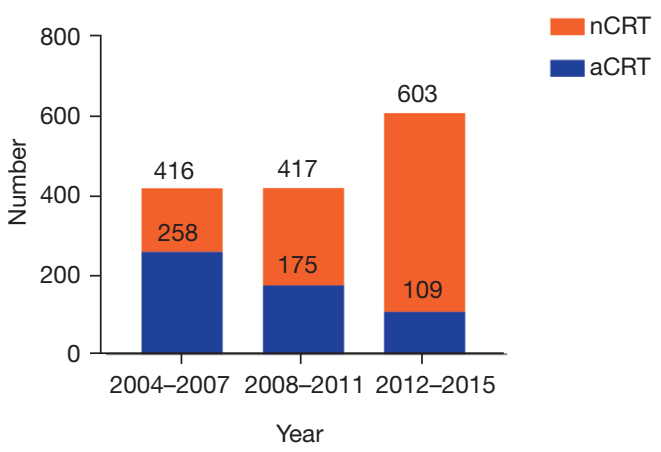

Figure 3 The number of patients receiving nCRT and aCRT in different time periods. nCRT, neoadjuvant chemoradiotherapy; aCRT, adjuvant chemoradiotherapy.

In the matched cohort, among patients with N1 disease, those who underwent nCRT had a more favourable OS than those who received aCRT [median OS: 35.0 months (95\% CI: 28.7-41.3 months) and 26 months (95\% CI: 22.8-29.2 months), respectively].

Moreover, we performed a subgroup analysis to investigate the survival outcomes and clinical benefit of nCRT and aCRT in patients with different T-stages (Figure 7). However, there were no statistically significant differences in the OS between the two cohorts based on $\mathrm{T}$ stage. There were also no significant survival benefits for the IVA stage, N2 stage, and N3 stage subgroups due to their small sample sizes.

\section{Discussion}

The current comprehensive treatment model from AEG comes from the treatment models of oesophageal adenocarcinoma and proximal gastric adenocarcinoma. However, AEG differs epidemiologically and biologically from these two types of cancer; therefore, it is still controversial whether these treatment regimens will benefit patients with AEG. And the domestic research on AEG currently focuses on the selection of different types of surgical methods, related research on immune and targeted therapy, and the comparison of neoadjuvant radiotherapy and chemotherapy with simple surgery, but there is a lack of comparative research on the two treatment methods (18-21). This study analysed the survival outcomes of patients with locally advanced AEG using the SEER database. After PSM, remarkable improvements were observed in the survival of patients undergoing nCRT. Similar survival benefits were reported in the meta-analyses by Valentini et al. (22) and Guo et al. (23), which compared the outcomes of patients undergoing surgery alone with those undergoing preoperative radiotherapy + surgery or those undergoing surgery + postoperative radiotherapy. These studies found that preoperative radiotherapy could significantly improve the 1-, 3-, 5-, and 10-year OS rates compared with surgery alone, but there was no survival benefit of postoperative radiotherapy, and the 5 -year survival rate in that group was even lower than that of the surgery alone group.

All patients in this study had stage IIIB or IVA disease consistent with the definition of locally advanced AEG and the $8^{\text {th }}$ edition of the TNM staging system. Our subgroup analysis indicated that the median OS was prolonged in patients who underwent nCRT. Furthermore, the survival benefit was greater in patients with more advanced tumours, larger tumours, later $\mathrm{T}$ stage, resection other than $\mathrm{R} 0$, and palliative surgical resection. Resection was found to be more likely to be successful in advanced tumours after nCRT, which increases survival; hence, nCRT is recommended for AEG patients with stage IIIB disease. However, there was no difference in survival between the two treatment methods in the stage IVA subgroup. There are several potential reasons for these findings. Firstly, preoperative tumor blood supply is complete, which is conducive to maintaining the local chemotherapy drug intensity and oxygen concentration of target lesions. In addition, nCRT can reduce the tumor volume, reduce the tumor stage and achieve R0 resection. Furthermore, nCRT has certain advantages in preventing postoperative recurrence, because it reduces intraoperative tumor implantation and metastasis and eliminates subclinical lesions and micrometastasis. Secondly, patients with stage IVA disease have advanced tumours and poor physical condition. thus, the treatment effect is not good regardless of whether they receive nCRT or aCRT. And after PSM, we had only 84 cases in this group, which may have been insufficient to ascertain the treatment effect. Furthermore, the SEER database provides no information on side effects after treatment and could not be used to further evaluate the treatment effect. Therefore, more randomized controlled studies for stage IVA AEG are needed.

We also performed subgroup analysis based on $\mathrm{N}$ stage. Patients with N1 disease had a survival benefit with nCRT, but there was no significant difference in survival between patients receiving nCRT and aCRT in the N2 and N3 subgroups. The INT-0116 study $(15,16)$ and the ARTIST study $(24,25)$ found similar results. These findings indicate that patients with lymph node metastasis 
Table 2 Patient characteristics in the propensity score-matched cohort

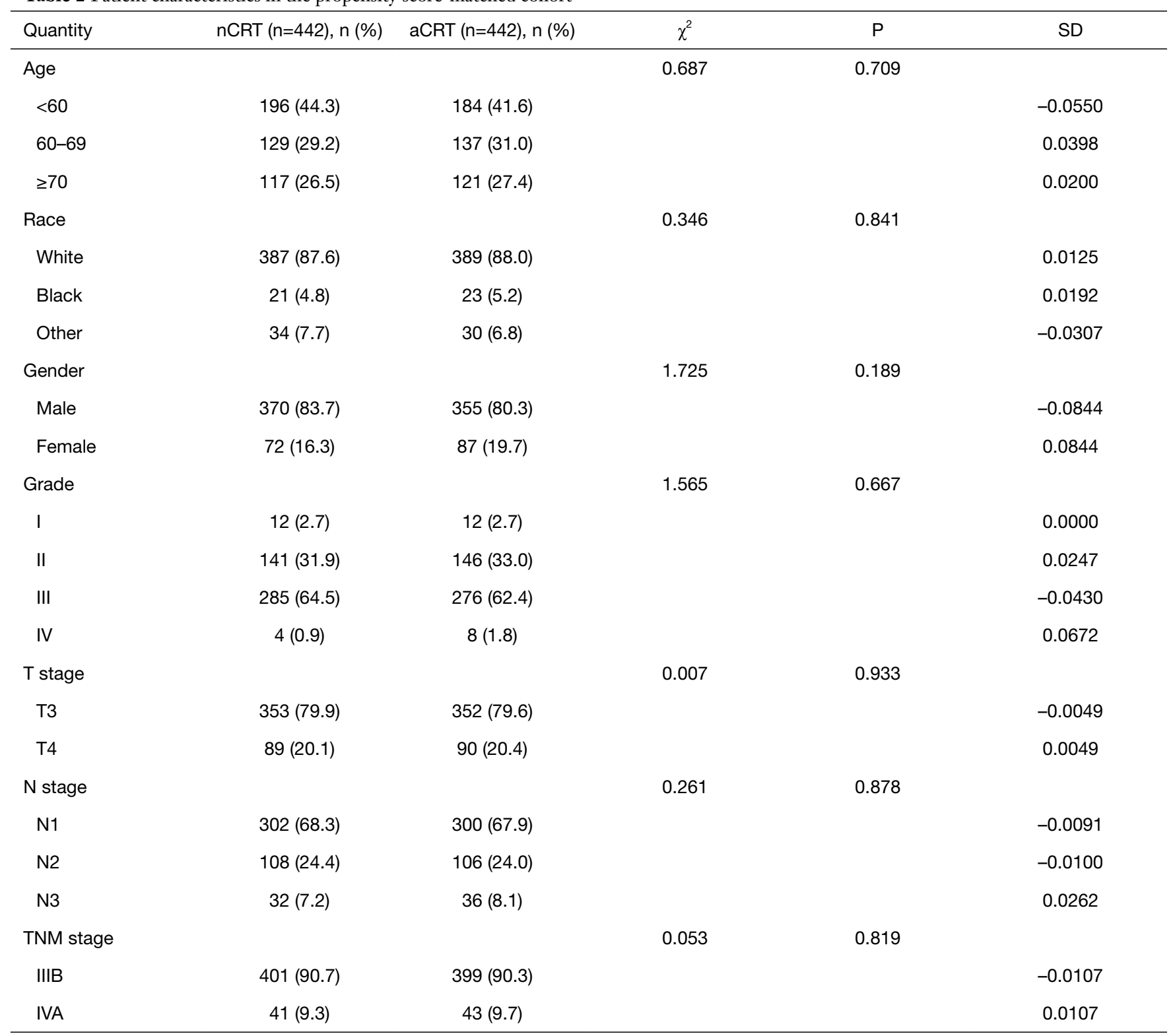

The Chi square test was applied to contrast patient and cancer characteristics in the pre-matched. Statistical significance was set at $\mathrm{P}<0.05$. nCRT, neoadjuvant chemoradiotherapy; aCRT, adjuvant chemoradiotherapy; SD, standardized differences.

can benefit from radiotherapy; however, patients whose cancer cells have metastasized in lymph nodes in advanced stage group (N2 and N3 stages) have no significant survival benefits. After PSM, we had only 214 patients with N2 stage and 68 patients with $\mathrm{N} 3$ stage, so this result may not be generalizable. However, the ARTIST Phase III trial $(26,27)$ found that aCRT does not reduce the recurrence rate in patients receiving $\mathrm{D} 2$ lymph node dissection, which further indicates that the benefits of aCRT are closely related to the surgical method. The SEER database does not provide information on $\mathrm{R} 0$ resection, surgical lymph node dissection, or postoperative pathological staging, and thus, the advantages of nCRT could not be further assessed based on these characteristics. Therefore, more prospective randomized clinical trials combined with surgical procedures during follow-up are necessary. 
A

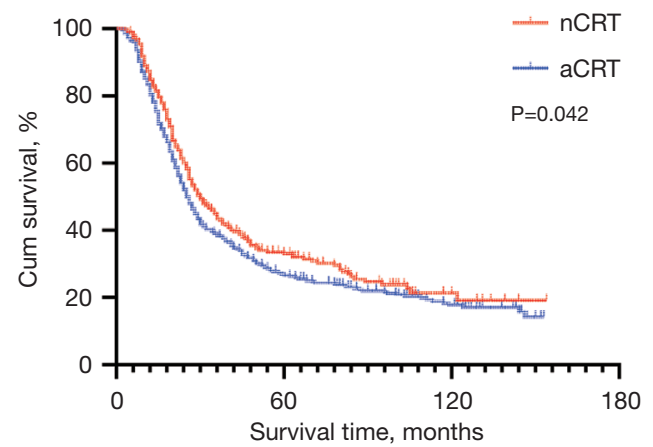

B Cancer specific-survival $(n=884)$

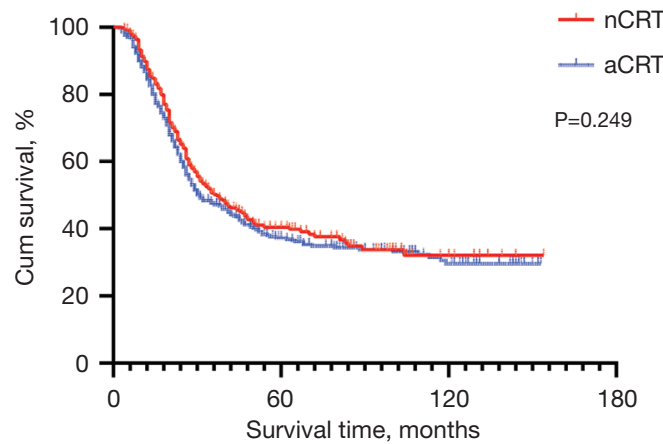

Figure 4 Survival curves for OS and CSS in patients with locally advanced adenocarcinoma of the oesophagogastric junction receiving nCRT and aCRT by Kaplan-Meier. (A) Overall survival; (B) cancer-specific survival. OS, overall survival; CCS, cancer-specific survival; nCRT, neoadjuvant chemoradiotherapy; aCRT, adjuvant chemoradiotherapy.

Table 3 Prognostic factors for overall survival

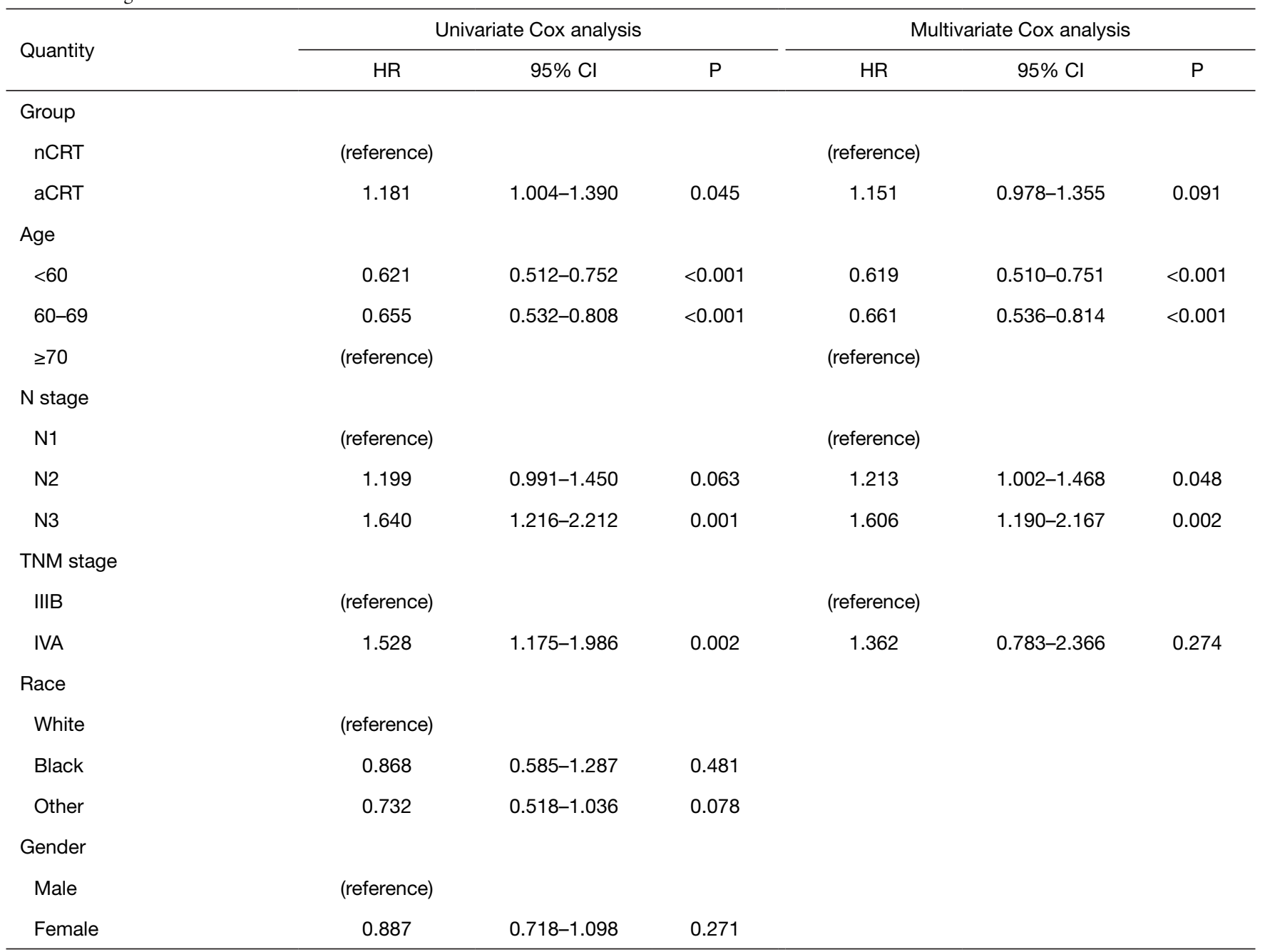

Table 3 (continued) 
Table 3 (continued)

\begin{tabular}{|c|c|c|c|c|c|c|}
\hline Quantity & \multicolumn{3}{|c|}{ Univariate Cox analysis } & \multicolumn{3}{|c|}{ Multivariate Cox analysis } \\
\hline \multicolumn{7}{|l|}{ Grade } \\
\hline 1 & (reference) & & & (reference) & & \\
\hline II & 0.868 & $0.565-1.619$ & 0.868 & & & \\
\hline IV & 0.158 & $0.793-4.147$ & 0.158 & & & \\
\hline \multicolumn{7}{|l|}{ T stage } \\
\hline T3 & (reference) & & & & & \\
\hline $\mathrm{T} 4$ & 1.027 & $0.848-1.244$ & 0.783 & & & \\
\hline
\end{tabular}

After matching, we employed Cox regression for univariate and multivariate analysis to determine the independent predictors in locally advanced AEG patients. The results are presented as HR with $95 \% \mathrm{Cl}$ (unless otherwise indicated). Double-tailed $\mathrm{P}$ values $<0.05$ were considered statistically significant. $\mathrm{nCRT}$, neoadjuvant chemoradiotherapy; aCRT, adjuvant chemoradiotherapy.

Table 4 Prognostic factors for cancer-specific survival

\begin{tabular}{|c|c|c|c|c|c|c|}
\hline Quantity & \multicolumn{3}{|c|}{ Univariate Cox analysis } & \multicolumn{3}{|c|}{ Multivariate Cox analysis } \\
\hline \multicolumn{7}{|l|}{ Group } \\
\hline $\mathrm{nCRT}$ & (reference) & & & (reference) & & \\
\hline aCRT & 1.113 & $0.926-1.338$ & 0.254 & 1.091 & $0.907-1.313$ & 0.353 \\
\hline$<60$ & 0.986 & $0.795-1.223$ & 0.898 & 0.903 & $0.716-1.140$ & 0.391 \\
\hline $60-69$ & 1.148 & $0.914-1.443$ & 0.235 & 0.849 & $0.662-1.090$ & 0.200 \\
\hline$\geq 70$ & (reference) & & & (reference) & & \\
\hline \multicolumn{7}{|l|}{ N stage } \\
\hline N3 & 1.888 & $1.372-2.597$ & $<0.001$ & 1.312 & $0.668-2.578$ & 0.430 \\
\hline \multicolumn{7}{|c|}{ TNM stage } \\
\hline IIIB & (reference) & & & (reference) & & \\
\hline IVA & 1.742 & $1.315-2.307$ & $<0.001$ & 1.778 & $1.341-2.359$ & $<0.001$ \\
\hline \multicolumn{7}{|l|}{ Race } \\
\hline White & (reference) & & & (reference) & & \\
\hline Black & 0.964 & $0.628-1.480$ & 0.867 & 0.994 & $0.646-1.528$ & 0.977 \\
\hline Other & 0.790 & $0.539-1.158$ & 0.227 & 0.757 & $0.515-1.113$ & 0.157 \\
\hline
\end{tabular}

Table 4 (continued) 
Table 4 (continued)

\begin{tabular}{|c|c|c|c|c|c|c|}
\hline Quantity & \multicolumn{3}{|c|}{ Univariate Cox analysis } & \multicolumn{3}{|c|}{ Multivariate Cox analysis } \\
\hline \multicolumn{7}{|l|}{ Gender } \\
\hline Male & (reference) & & & (reference) & & \\
\hline Female & 0.927 & $0.730-1.177$ & 0.533 & 0.886 & $0.694-1.130$ & 0.329 \\
\hline 1 & (reference) & & & (reference) & & \\
\hline II & 1.191 & $0.606-2.340$ & 0.612 & 1.236 & $0.629-2.429$ & 0.539 \\
\hline III & 1.631 & $0.841-3.166$ & 0.148 & 1.717 & $0.884-3.334$ & 0.111 \\
\hline IV & 2.398 & $0.892-6.444$ & 0.083 & 2.284 & $0.849-6.141$ & 0.102 \\
\hline T4 & 1.025 & $0.823-1.277$ & 0.824 & 1.082 & $0.846-1.383$ & 0.530 \\
\hline
\end{tabular}

After matching, we employed Cox regression for univariate and multivariate analysis to determine the independent predictors in locally advanced AEG patients. The results are presented as HR with $95 \% \mathrm{Cl}$ (unless otherwise indicated). Double-tailed $\mathrm{P}$ values $<0.05$ were considered statistically significant. nCRT, neoadjuvant chemoradiotherapy; aCRT, adjuvant chemoradiotherapy.
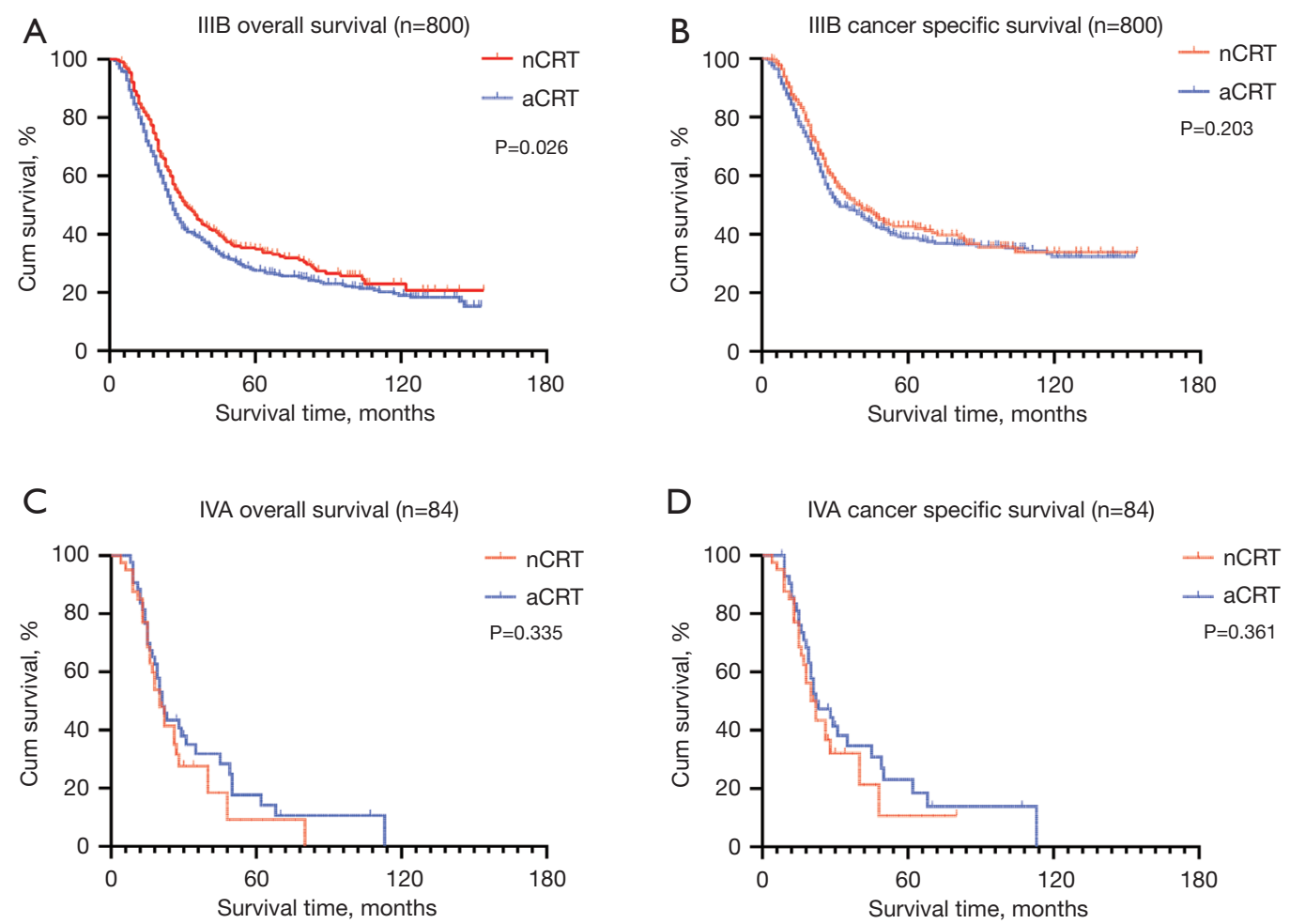

Figure 5 OS and CSS survival curves constructed using the Kaplan-Meier method in patients with locally advanced AEG receiving nCRT and aCRT based on the TNM stage. (A) Stage IIIB, overall survival; (B) stage IIIB, cancer-specific survival; (C) stage IVA, overall survival; (D) stage IVA, cancer-specific survival. OS, overall survival; CCS, cancer-specific survival; AEG, adenocarcinoma of the oesophagogastric junction; nCRT, neoadjuvant chemoradiotherapy; aCRT, adjuvant chemoradiotherapy. 

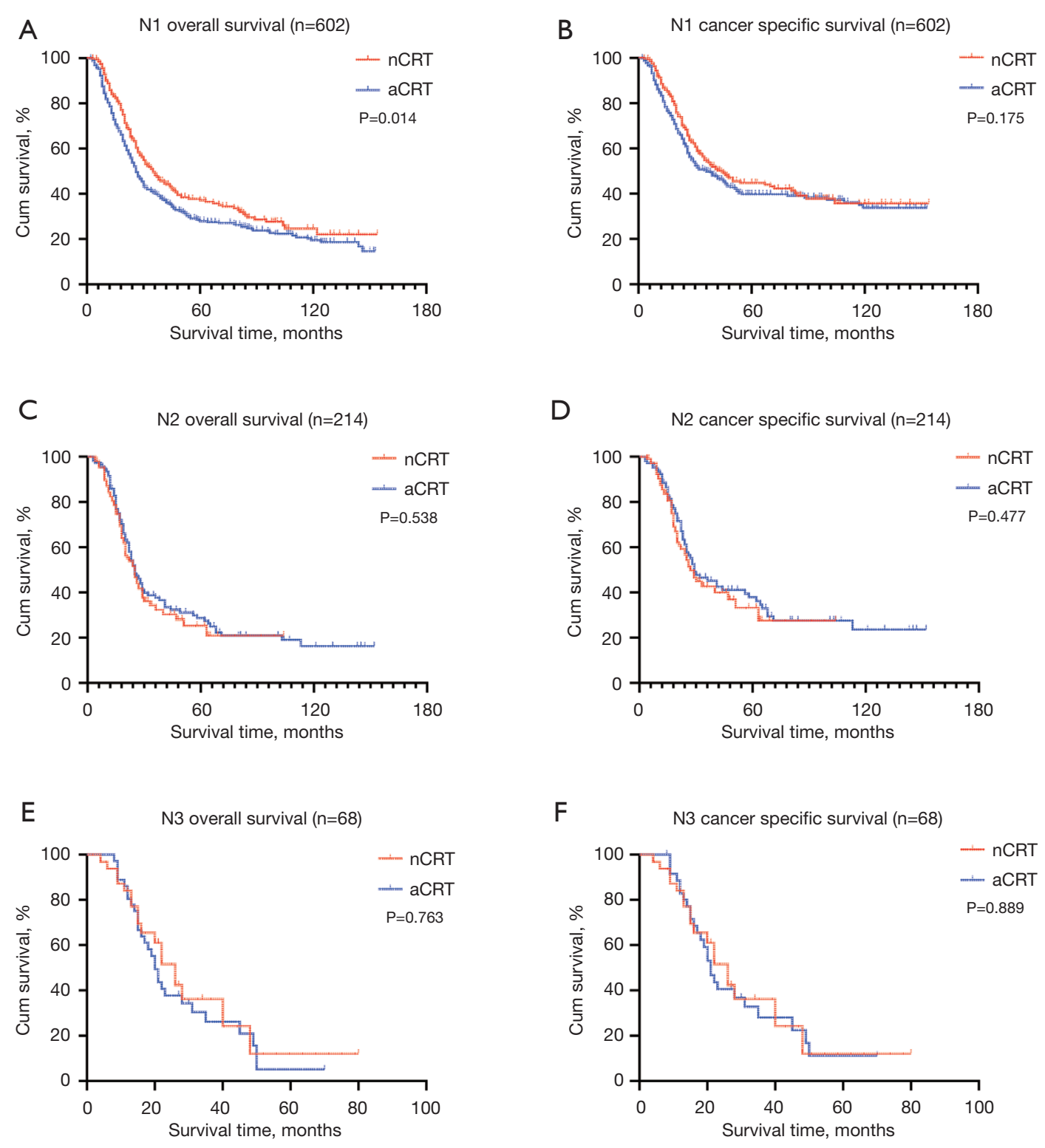

Figure 6 OS and CSS survival curves constructed using the Kaplan-Meier method in patients with locally advanced AEG receiving nCRT and aCRT based on the N stage. (A) N1 stage, overall survival; (B) N1 stage, cancer-specific survival; (C) N2 stage, overall survival; (D) N2 stage, cancer-specific survival; (E) N3 stage, overall survival; (F) N3 stage, cancer-specific survival. OS, overall survival; CCS, cancerspecific survival; AEG, adenocarcinoma of the oesophagogastric junction; nCRT, neoadjuvant chemoradiotherapy; aCRT, adjuvant chemoradiotherapy.

The strength of this study is the large sample size and the PSM analysis. The use of PSM to reduce the bias in terms of age, race, sex, grade, total stage, $\mathrm{T}$ stage, and $\mathrm{N}$ stage is necessary for pre- and post-matching chi-square tests and standardized difference comparisons. Although the propensity score method does not completely eliminate confounding variables (28), it is often more practical and statistically valid than multivariate statistical methods in observational studies (29). Also, PSM is a good substitute when RCT data is unavailable (30).

However, there are some limitations in this study that should be noted. Being a retrospective study, potential selection bias was inevitable. Furthermore, there were still unadjusted confounding factors even after PSM, and the 

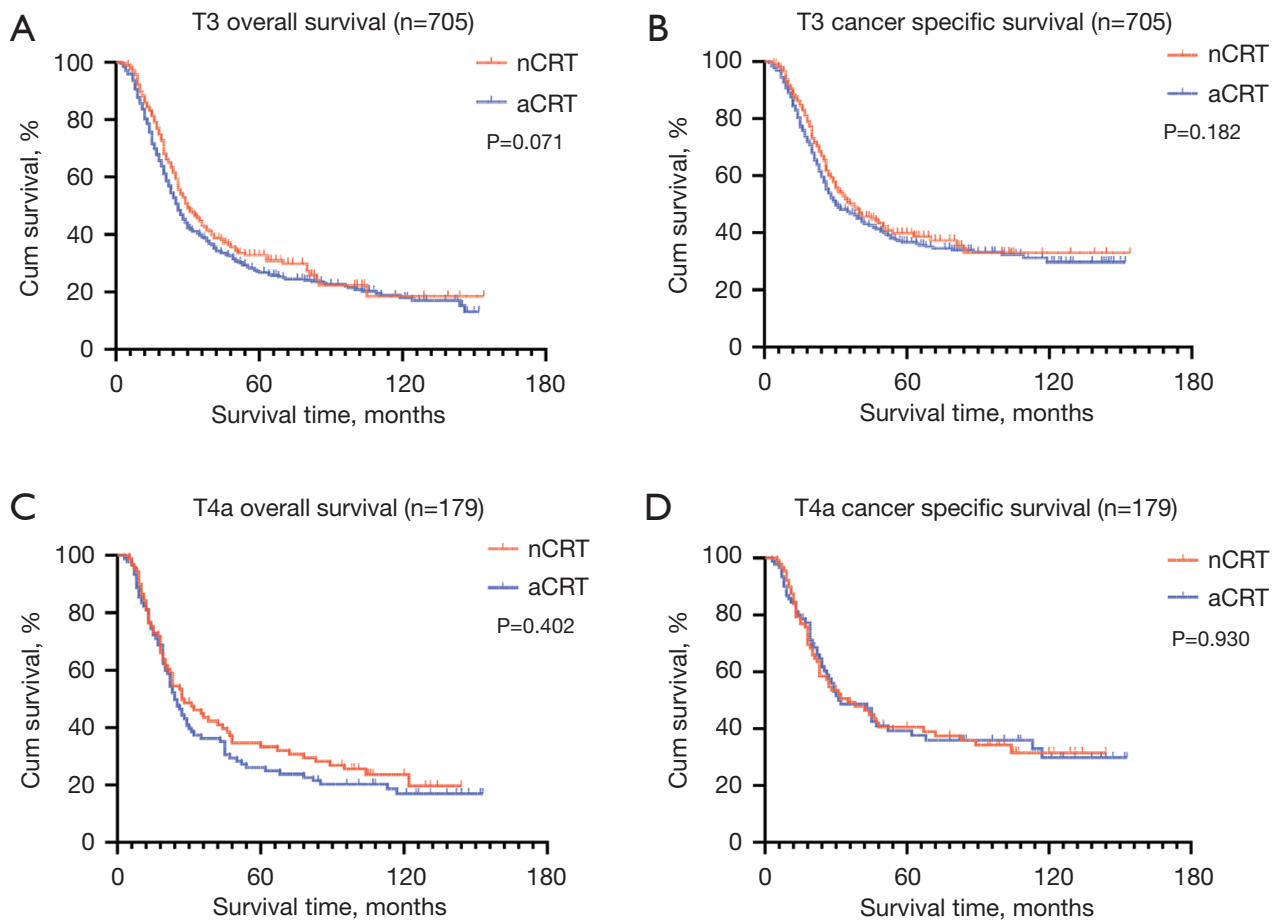

Figure 7 OS and CSS survival curves constructed using the Kaplan-Meier method in patients with locally advanced AEG in patients receiving nCRT and aCRT based on the T stage. (A) T3 stage, overall survival; (B) T3 stage, cancer-specific survival; (C) T4a stage, overall survival; (D) T4a stage, cancer-specific survival. OS, overall survival; CCS, cancer-specific survival; AEG, adenocarcinoma of the oesophagogastric junction; nCRT, neoadjuvant chemoradiotherapy; aCRT, adjuvant chemoradiotherapy.

SEER database does not provide more detailed information, such as surgical methods, gene mutation status, recurrence, underlying diseases and comorbidities, and non-systemic chemotherapy regimens, which are important factors affecting the prognosis of AEG. In addition, SEER lacks information on the different methods and specific parameters of radiation therapy (such as the dose, segmentation size, exposure range, and radiation technology), all of which may affect patient prognosis. However, despite its limitations, the relatively large sample size and the use of the PSM method may reduce these biases to a certain extent.

\section{Conclusions}

Surgery-based nCRT significantly prolonged the survival of patients with locally advanced AEG, especially those with stage IIIB and N1 after carefully selected.

\section{Acknowledgments}

Funding: None.

\section{Footnote}

Reporting Checklist: The authors have completed the STROBE reporting checklist. Available at https://jgo. amegroups.com/article/view/10.21037/jgo-21-815/rc

Conflicts of Interest: All authors have completed the ICMJE uniform disclosure form (available at https://jgo.amegroups. com/article/view/10.21037/jgo-21-815/coif). The authors have no conflicts of interest to declare.

Ethical Statement: The authors are accountable for all aspects of the work in ensuring that questions related to the accuracy or integrity of any part of the work are appropriately investigated and resolved. The study was conducted in accordance with the Declaration of Helsinki (as revised in 2013).

Open Access Statement: This is an Open Access article distributed in accordance with the Creative Commons Attribution-NonCommercial-NoDerivs 4.0 International 
License (CC BY-NC-ND 4.0), which permits the noncommercial replication and distribution of the article with the strict proviso that no changes or edits are made and the original work is properly cited (including links to both the formal publication through the relevant DOI and the license). See: https://creativecommons.org/licenses/by-nc-nd/4.0/.

\section{References}

1. Cao W, Chen HD, Yu YW, et al. Changing profiles of cancer burden worldwide and in China: a secondary analysis of the global cancer statistics 2020. Chin Med J (Engl) 2021;134:783-91.

2. Wu JD, Huang Y, Fang CY, et al. Progress in comprehensive treatment of locally advanced esophageal gastric junction adenocarcinoma. Chinese Journal of Clinical Thoracic and Cardiovascular Surgery 2021.

3. Colquhoun A, Arnold M, Ferlay J, et al. Global patterns of cardia and non-cardia gastric cancer incidence in 2012. Gut 2015;64:1881-8.

4. Imamura $Y$, Watanabe $M$, Toihata $T$, et al. Recent Incidence Trend of Surgically Resected Esophagogastric Junction Adenocarcinoma and Microsatellite Instability Status in Japanese Patients. Digestion 2019;99:6-13.

5. Liu K, Yang K, Zhang W, et al. Changes of Esophagogastric Junctional Adenocarcinoma and Gastroesophageal Reflux Disease Among Surgical Patients During 1988-2012: A Single-institution, High-volume Experience in China. Ann Surg 2016;263:88-95.

6. Siewert JR, Hölscher AH, Becker K, et al. Cardia cancer: attempt at a therapeu-tically relevant classification. Chirurg 1987;58:25-32.

7. Yuasa $N$, Miyake H, Yamada $T$, et al. Clinicopathologic comparison of Siewert type II and III adenocarcinomas of the gastroesophageal junction. World J Surg 2006;30:364-71.

8. Hasegawa $\mathrm{S}$, Yoshikawa $\mathrm{T}, \mathrm{Cho} \mathrm{H}$, et al. Is adenocarcinoma of the esophagogastric junction different between Japan and western countries? The incidence and clinicopathological features at a Japanese high-volume cancer center. World J Surg 2009;33:95-103.

9. Siewert JR, Feith M, Stein HJ. Biologic and clinical variations of adenocarcinoma at the esophago-gastric junction: relevance of a topographic-anatomic subclassification. J Surg Oncol 2005;90:139-46; discussion 146.

10. Zhang Z, Li G. Clinical value of neoadjuvant chemoradiotherapy in the treatment of locally advanced gastric cancer. Chinese Journal of Practical Surgery
2017;37:1109-14.

11. Omloo JM, Lagarde SM, Hulscher JB, et al. Extended transthoracic resection compared with limited transhiatal resection for adenocarcinoma of the mid/distal esophagus: five-year survival of a randomized clinical trial. Ann Surg 2007;246:992-1000; discussion 1000-1.

12. Sasako M, Sano T, Yamamoto S, et al. Left thoracoabdominal approach versus abdominal-transhiatal approach for gastric cancer of the cardia or subcardia: a random-ised controlled trial. Lancet Oncol 2006;7:644-51.

13. Shapiro J, van Lanschot JJB, Hulshof MCCM, et al. Neoadjuvant chemoradio-therapy plus surgery versus surgery alone for oesophageal or junctional cancer (CROSS): long-term results of a randomised controlled trial. Lancet Oncol 2015;16:1090-8.

14. Sasako M, Sakuramoto S, Katai H, et al. Five-year outcomes of a randomized phase III trial comparing adjuvant chemotherapy with S-1 versus surgery alone in stage II or III gastric cancer. J Clin Oncol 2011;29:4387-93.

15. Bleiberg H, Goffin JC, Dalesio O, et al. Adjuvant radiotherapy and chemotherapy in resectable gastric cancer. A randomized trial of the gastro-intestinal tract cancer co-operative group of the EORTC. Eur J Surg Oncol 1989;15:535-43.

16. von Döbeln GA, Klevebro F, Jacobsen AB, et al. Neoadjuvant chemotherapy versus neoadjuvant chemoradiotherapy for cancer of the esophagus or gastroesophageal junction: long-term results of a randomized clinical trial. Dis Esophagus 2019. doi: 10.1093/dote/doy078.

17. Zhao QY, Luo JC, Su Y, et al. Propensity score matching with R: conventional methods and new features. Ann Transl Med 2021;9:812.

18. Luo SJ, Xiong WW, Jun Chen Y, et al. Application of thoracic single hole assisted laparoscopic "five step" mediastinal lymph node dissection in Siewert ii esophageal gastric junction adenocarcinoma. Zhonghua Wei Chang Wai Ke Za Zhi 2021;24:684-90.

19. Li T, Liu L, Tao R, et al. Advances in targeted therapy and immunotherapy for locally advanced or advanced gastric cancer or gastric-esophageal junction cancer and the exploration of biomarkers. Journal of Digestive Oncology (Electronic Version) 2021;13:216-22.

20. Tian Y, Wang Q, Wang J, et al. Long term prognosis of a randomized controlled trial of neoadjuvant concurrent chemoradiotherapy combined with surgery in the treatment of Siewert type II and III esophageal gastric junction adenocarcinoma. Chinese Journal of 
Gastrointestinal Surgery 2021;24:128-37.

21. Wang J, Zhao Q, Huang X, et al. Feasibility of intensity-modulated radia-tion therapy with concurrent chemotherapy in patients with Siewert type II and III ade-nocarcinoma of the esophagogastric junction before surgery. Chinese Journal of Radiation Oncology 2017;26:1269-75.

22. Valentini V, Cellini F, Minsky BD, et al. Survival after radiotherapy in gastric cancer: systematic review and metaanalysis. Radiother Oncol 2009;92:176-83.

23. Guo L, Wang X, Ma B, et al. Radiotherapy combined with surgical treatment for gastric cancer: a meta-analysis. Chin Ger J Clin Oncol 2011;10:442.

24. Lee J, Lim DH, Kim S, et al. Phase III trial comparing capecitabine plus cisplatin versus capecitabine plus cisplatin with concurrent capecitabine radiotherapy in com-pletely resected gastric cancer with D2 lymph node dissection: the ARTIST trial. J Clin Oncol 2012;30:268-73.

25. Park SH, Sohn TS, Lee J, et al. Phase III Trial to Compare Adjuvant Chemotherapy With Capecitabine and Cisplatin Versus Concurrent Chemoradiotherapy in Gastric Cancer: Final Report of the Adjuvant Chemoradiotherapy in Stomach Tumors Trial, In-cluding Survival and Subset Analyses. J Clin Oncol 2015;33:3130-6.

Cite this article as: Zhang F, Feng X, Li Y, Yan J, Zhang Z, Song X. Survival outcomes of neoadjuvant and adjuvant chemoradiotherapy for locally advanced adenocarcinoma of the oesophagogastric junction: a retrospective cohort study using the SEER database. J Gastrointest Oncol 2022;13(1):26-39. doi: 10.21037/jgo-21-815
26. Al-Batran SE, Hofheinz RD, Pauligk C, et al.

Histopathological regression after neoadjuvant docetaxel, oxaliplatin, fluorouracil, and leucovorin versus epirubicin, cis-platin, and fluorouracil or capecitabine in patients with resectable gastric or gas-tro-oesophageal junction adenocarcinoma (FLOT4-AIO): results from the phase 2 part of a multicentre, open-label, randomised phase 2/3 trial. Lancet Oncol 2016;17:1697-708.

27. Cai Z, Yin Y, Shen C, et al. Comparative effectiveness of preoperative, postop-erative and perioperative treatments for resectable gastric cancer: A network me-ta-analysis of the literature from the past 20 years. Surg Oncol 2018;27:563-74.

28. Bosco JL, Silliman RA, Thwin SS, et al. A most stubborn bias: no adjustment method fully resolves confounding by indication in observational studies. J Clin Epidemiol 2010;63:64-74.

29. Haukoos JS, Lewis RJ. The Propensity Score. JAMA 2015;314:1637-8.

30. Austin PC. An Introduction to Propensity Score Methods for Reducing the Effects of Confounding in Observational Studies. Multivariate Behav Res 2011;46:399-424.

(English Language Editor: A. Kassem) 